\title{
POSTOPERATIVE ATRIAL FIBRILLATION PROPHYLAXIS AND LUNG RESECTION - OUR EXPERIENCE WITH 608 CONSECUTIVE PATIENTS
}

\author{
Vjekoslav Karadža, Dinko Stančić-Rokotov, Jasna Špiček Macan, Nevenka Hodoba, \\ Nevenka Kolarić and Sanja Sakan
}

Jordanovac Department of Thoracic Surgery, Zagreb University Hospital Center, Zagreb, Croatia

SUMMARY - Postoperative atrial fibrillation is a common complication after lung resection. It is burdened by increased mortality and morbidity, prolonged hospitalization, and higher resource utilization in thoracic surgery patients. Therefore, some kind of pharmacological prophylaxis is recommended. In our patients, diltiazem, a calcium antagonist, is administered. We collected data on all 608 patients having undergone lung resection (no less than lobectomy) between November 2012 and May 2015. This period included patients having received diltiazem during their postoperative stay in our Intensive Care Unit and surgical ward, and those that did not receive it. Patients having had atrial fibrillation before the surgery and patients with cardiac pacemaker were excluded from the trial. Other patients were divided into three groups: patients with some kind of antiarrhythmic therapy before and continued after the surgery; patients with diltiazem prophylaxis; and patients without any antiarrhythmic prophylaxis. The data collected were statistically analyzed. We found no statistically significant difference in the incidence of postoperative atrial fibrillation among the groups $(\mathrm{p}<0.05)$.

Key words: Diltiazem; Atrial fibrillation; Thoracic surgery

\section{Introduction}

According to the literature, postoperative atrial fibrillation (POAF) (or equivalent supraventricular arrhythmia) is a common complication after pulmonary and esophageal surgery, with the incidence between $12 \%$ and $44 \% \%^{1,2}$. Some risk factors have been identified, correlation with perioperative mortality and morbidity has been demonstrated, and prophylactic and therapeutic guidelines have been developed ${ }^{1,2}$. POAF is associated with hemodynamic instability, heart failure, thromboembolic events, higher resource utilization and, possibly, increased mortality ${ }^{1}$. The precise mechanism of POAF development is not well understood $^{1}$. The risk factors include history of smoking, obesity, advancing age, atrial dilatation, myocardial

Correspondence to: Vjekoslav Karadža, MD, Jordanovac Department of Thoracic Surgery, Zagreb University Hospital Center, Jordanovac 104, HR-10000 Zagreb, Croatia

E-mail: vkaradza@xnet.hr, anestezija.tk3@kbc-zagreb.hr

Received November 10, 2015, accepted September 28, 2016 ischemia, volume overload, history of heart failure, increased adrenergic and vagal tone, and surgical procedures associated with local or systemic inflammation ${ }^{1,2}$. The extent of pulmonary resection is of importance as a risk factor, with the highest rates of incidence noted after pneumonectomies, extrapleural pneumonectomies and lung transplantations ${ }^{2}$. Lobectomies and pneumonectomies are recognized as high risk procedures with POAF incidence above 15\% ${ }^{1}$. Among drugs recommended for the prophylaxis of POAF and equivalent postoperative arrhythmias is diltiazem, a calcium antagonist ${ }^{2,3}$. It has few side effects and, with respect to known contraindications, it is well tolerated, especially in patients with chronic obstructive pulmonary disease $(\mathrm{COPD})^{2}$.

\section{Patients and Methods}

This observational study was performed at Jordanovac Department of Thoracic Surgery, Zagreb University Hospital Centre, Zagreb, Croatia. Data from med- 
Table 1. Extent of lung resection

\begin{tabular}{|l|c|c|}
\hline Frequency table \\
\hline Lung resection & $\mathrm{n}$ & $\%$ \\
\hline PD & 26 & 4.4 \\
PS & 56 & 9.5 \\
BID & 27 & 4.6 \\
BSD & 16 & 2.7 \\
LID & 77 & 13.1 \\
LSS & 121 & 20.5 \\
LIS & 82 & 13.9 \\
LSD & 149 & 25.3 \\
LMD & 35 & 5.9 \\
Total & 589 & 100.0 \\
\hline
\end{tabular}

$\mathrm{PD}=$ right pneumonectomy; $\mathrm{PS}=$ left pneumonectomy; $\mathrm{BID}=$ right inferior bilobectomy; $\mathrm{BSD}=$ right superior bilobectomy; $\mathrm{LID}$ = right inferior lobectomy; LSS = left superior lobectomy; LIS = left inferior lobectomy; LSD = right superior lobectomy; LMD = right middle lobe resection

Table 2. Incidence of atrial fibrillation $(A F)$

\begin{tabular}{|l|c|c|}
\hline Frequency table \\
\hline $\mathrm{AF}$ & $\mathrm{n}$ & $\%$ \\
\hline Yes & 77 & 13.1 \\
No & 512 & 86.9 \\
Total & 589 & 100.0 \\
\hline
\end{tabular}

Table 3. Prophylaxis groups

\begin{tabular}{|l|c|c|}
\hline Frequency table \\
\hline Atrial fibrillation prophylaxis & $\mathrm{n}$ & $\%$ \\
\hline Diltiazem & 221 & 37.5 \\
Preoperative antiarrhythmic therapy & 132 & 22.4 \\
None & 236 & 40.1 \\
Total & 589 & 100.0 \\
\hline
\end{tabular}

ical records of all patients having undergone pulmonary resection procedures with a high risk of $\mathrm{POAF}^{1}$ (lobectomies, bilobectomies and pneumonectomies) between November 2012 and May 2015 were included in the study. It was the time of introducing diltiazem for atrial fibrillation (AF) prophylaxis in our patients, thus including patient data before and during the introduction of diltiazem prophylaxis. The following data were collected: patient age, sex, the American
Society of Anesthesiologists Physical Status (ASA status), comorbidity (coronary disease, arterial hypertension, other cardiac diseases and arrhythmias, preoperative chemotherapy, preoperative irradiation, hypo-/ hyperthyroidism, diabetes mellitus, renal insufficiency, alcoholic cirrhosis, concomitant malignancy), relevant preoperative drug therapy, lung resection type (extent, intrapericardial, extrapleural), use/no use of thoracic epidural analgesia (TEA), use/no use of POAF prophylaxis (and if, what kind), presence/absence of postoperative fibrillation and equivalent arrhythmia, and the highest ventricular response recorded during POAF. The choice of risk factors and collected data was determined by the data available from medical records. All patients had spent at least one night, most of them two or more nights, in the Intensive Care Unit (ICU) before they were admitted to thoracic surgery ward. Patients were divided into three groups: group A included patients with no POAF prophylaxis; group B included patients with diltiazem prophylaxis; and group $\mathrm{C}$ included patients with preoperative antiarrhythmic therapy (mostly beta-blockers, a few propafenone or amiodarone) that was continued postoperatively. Diltiazem was included in therapy, if there were no contraindications, on the day of surgery or the first postoperative day (according to clinical circumstances) and continued after transfer from ICU to surgical ward. A patient was included in group B if the 24-hour diltiazem dose was at least $60 \mathrm{mg}$ (usually it was between $60 \mathrm{mg}$ and $240 \mathrm{mg}$ per os divided in 2-4 doses). All patients were treated equally according to the ICU and surgical ward perioperative treatment protocol. All patients gave their informed consent for participation in the study. Statistical analysis and data analysis were done by the licensed STATISTICA 6.1 StatSoft Inc. 1983-2003 (serial number AGA304B211928E61) software. The sample was presented by descriptive statistics and frequency tables. Correlation between the highest recorded ventricular frequency of $\mathrm{AF}$ and other parameters was tested by the analysis of variance (ANOVA) and t-test for independent samples. Correlation between the incidence of $\mathrm{AF}$ and other parameters was presented in contingency tables and tested by $\chi^{2}$-test. Results of statistical analysis were presented graphically and numerically (in tables). Statistical testing was performed at the level of significance of $95 \%(\alpha=0.05)$. 


\section{Results}

There were 608 patients undergoing lung resection greater or equal to lobectomy during the period of 31 months. As 15 patients with chronic AF and four pa- tients with some kind of pacemaker were excluded, there remained 589 patients with lung resection for further analysis (Table 1). Seventy-seven of these 589 patients developed POAF (13.1\%) (Table 2). There were 82 pneumonectomies with $19(23.17 \%)$ cases of

Table 4. Postoperative atrial fibrillation prophylaxis and incidence

\begin{tabular}{|l|l|l|l|l|}
\hline \multicolumn{6}{|l|}{ 2-way summary table: frequencies observed } \\
\hline AF & $\begin{array}{l}\text { AF prophylaxis: } \\
\text { diltiazem }\end{array}$ & $\begin{array}{l}\text { AF prophylaxis: preoperative } \\
\text { antiarrhythmic therapy }\end{array}$ & $\begin{array}{l}\text { AF prophylaxis: } \\
\text { none }\end{array}$ & Total \\
\hline Yes & 27 & 16 & 34 & $74.16 \%$ \\
$\%$ & $35.06 \%$ & $20.78 \%$ & 202 & 77 \\
\hline No & 194 & 116 & $39.45 \%$ & 512 \\
$\%$ & $37.89 \%$ & $22.66 \%$ & 236 & 589 \\
\hline Total & 221 & 132 & & \\
\hline
\end{tabular}

$\mathrm{AF}=$ atrial fibrillation

Table 5. Statistics: $P O A F$ and POAF prophylaxis $(A F)$

Statistics: $\mathrm{AF}(2) \mathrm{x}$ AF prophylaxis(3)

\begin{tabular}{|l|c|c|c|}
\hline & $\chi^{2}$-test & $\mathrm{df}$ & $\mathrm{p}$ \\
\hline Pearson $\chi^{2}$-test & 0.617 & 2 & 0.734 \\
\hline
\end{tabular}

$\mathrm{POAF}=$ postoperative atrial fibrillation; $\mathrm{AF}=$ atrial fibrillation
POAF. According to their AF prophylaxis, these 589 patients were divided into 3 groups: group A, 236 patients without prophylaxis; group B, 221 patients with diltiazem prophylaxis; and group C, 132 patients with continued preoperative antiarrhythmic therapy (Table 3). POAF was recorded in 34 (14.41\%) group

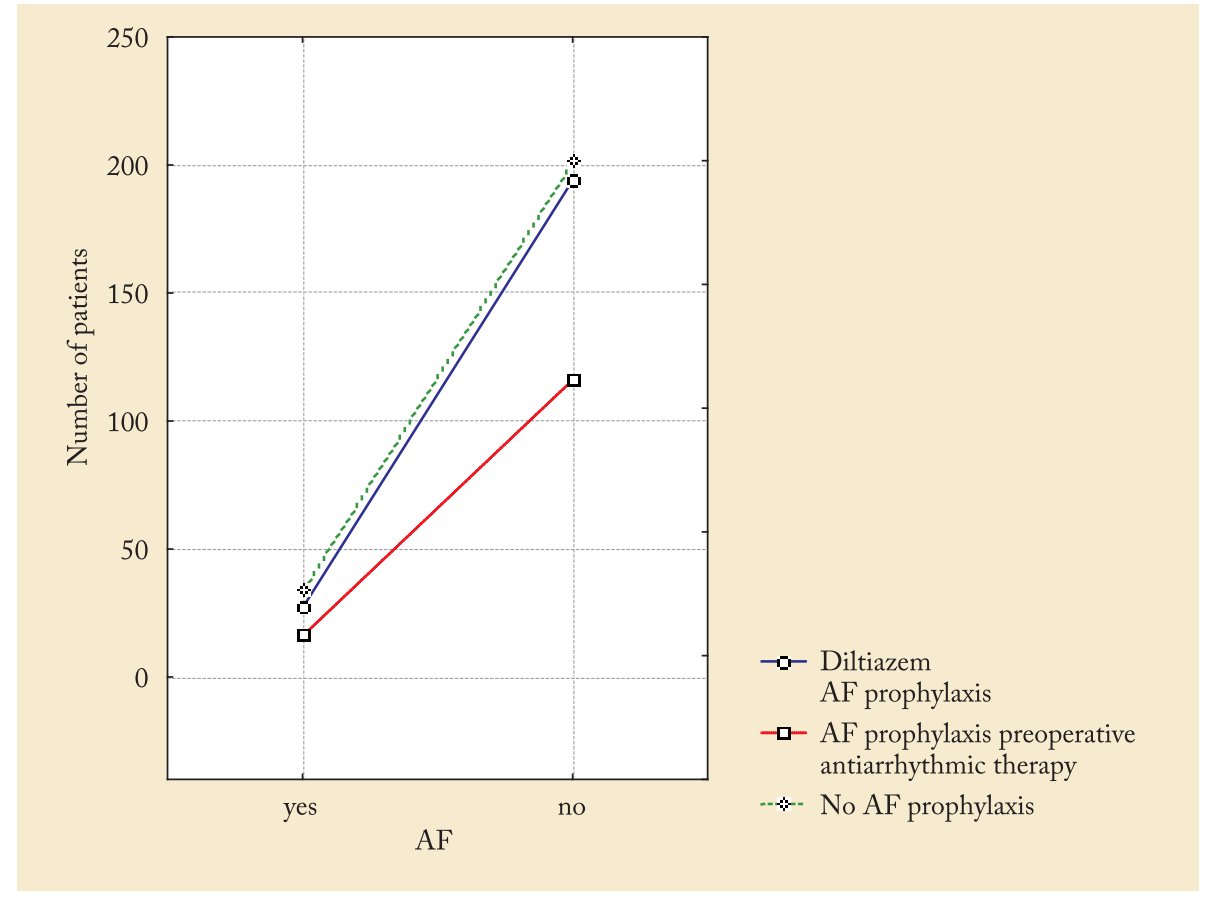

$\mathrm{POAF}=$ postoperative atrial fibrillation; $\mathrm{AF}=$ atrial fibrillation

Fig. 1. POAF prophylaxis and incidence. 
Table 6. $P O A F$ prophylaxis and $P O A F$ as the highest ventricular response recorded (here: $A F$ frequency)

\begin{tabular}{|l|l|l|l|l|l|l|}
\hline AF prophylaxis & N & $\begin{array}{l}\text { AF frequency } \\
\text { Mean }\end{array}$ & $\begin{array}{l}\text { AF frequency } \\
\text { SD }\end{array}$ & $\begin{array}{l}\text { AF frequency } \\
\text { SE }\end{array}$ & $\begin{array}{l}\text { AF frequency } \\
\text {-95\% CI }\end{array}$ & $\begin{array}{l}\text { AF frequency } \\
+95 \% \text { CI }\end{array}$ \\
\hline Total & 77 & 149.6234 & 22.79430 & 2.597651 & 144.4497 & 154.7970 \\
$\begin{array}{l}\text { Diltiazem } \\
\begin{array}{l}\text { Preoperative antiarrhythmic } \\
\text { therapy }\end{array}\end{array}$ & 27 & 148.1481 & 20.48208 & 3.941778 & 140.0457 & 156.2506 \\
None & 16 & 146.2500 & 14.24313 & 3.560782 & 138.6604 & 153.8396 \\
\hline
\end{tabular}

$\mathrm{POAF}=$ postoperative atrial fibrillation; $\mathrm{AF}=$ atrial fibrillation; $\mathrm{SD}=$ standard deviation; $\mathrm{SE}$ = standard error

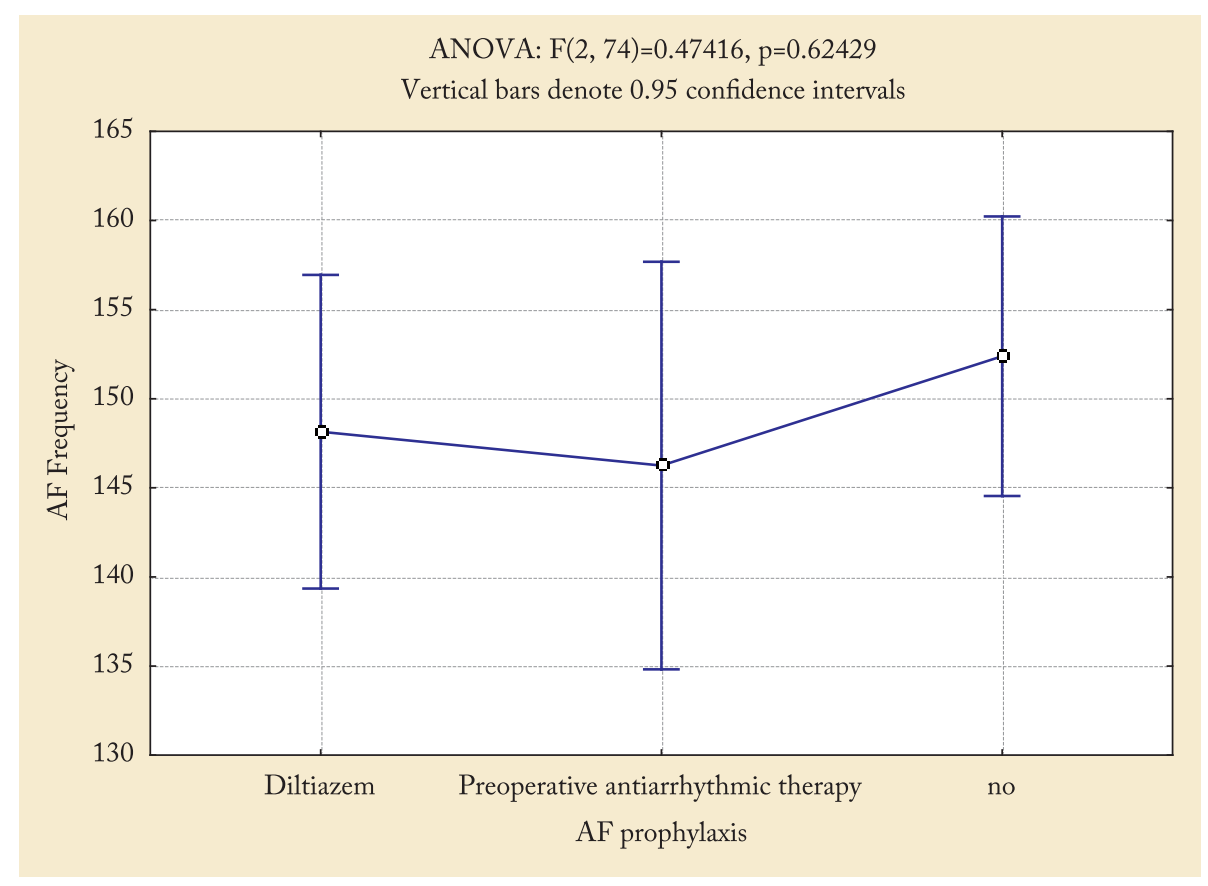

Fig. 2. Testing of the highest ventricular frequency observed and POAF prophylaxis.

A patients, 27 (12.22\%) group B patients and 16 (12.12\%) group $\mathrm{C}$ patients. The highest ventricular response in $\mathrm{AF}$ was recorded in each patient, with median 160 (range 91-230) in group A, 150 (range

Table 7. Age groups

\begin{tabular}{|l|c|c|}
\hline Frequency table & \\
\hline $\begin{array}{l}\text { Age group } \\
\text { (yrs) }\end{array}$ & $\mathrm{n}$ & $\%$ \\
\hline$\leq 55$ & 110 & 18.7 \\
$65-74$ & 190 & 32.3 \\
$56-64$ & 248 & 42.1 \\
$\geq 75$ & 41 & 7.0 \\
Total & 589 & 100.0 \\
\hline
\end{tabular}

Table 8. Age groups and POAF (AF)

\begin{tabular}{|l|c|c|c|}
\hline \multicolumn{4}{|l|}{ 2-way summary table: frequencies observed } \\
\hline $\begin{array}{l}\text { Age group } \\
\text { (yrs) }\end{array}$ & $\begin{array}{c}\mathrm{AF} \\
\text { yes }\end{array}$ & $\begin{array}{c}\mathrm{AF} \\
\text { no }\end{array}$ & Total \\
\hline$\leq 55$ & 9 & 101 & 110 \\
$\%$ & $8.18 \%$ & $91.82 \%$ & \\
$56-64$ & 27 & 221 & 248 \\
$\%$ & $10.89 \%$ & $89.11 \%$ & \\
$65-74$ & 30 & 160 & 190 \\
$\%$ & $15.79 \%$ & $84.21 \%$ & \\
$\geq 75$ & 11 & 30 & 41 \\
$\%$ & $26.83 \%$ & $73.17 \%$ & \\
\hline Total & 77 & 512 & 589 \\
\hline
\end{tabular}

$\mathrm{POAF}=$ postoperative atrial fibrillation; $\mathrm{AF}=$ atrial fibrillation 
Table 9. Statistics: age groups and POAF (AF)

\begin{tabular}{|l|c|c|c|}
\hline \multicolumn{4}{|l|}{ Statistics: age group(4) $\mathrm{AF}(2)$} \\
\hline & $\chi^{2}$-test & $\mathrm{df}$ & $\mathrm{p}$ \\
\hline Pearson $\chi^{2}$-test & 11.41967 & 3 & 0.00966 \\
\hline
\end{tabular}

$\mathrm{POAF}=$ postoperative atrial fibrillation; $\mathrm{AF}=$ atrial fibrillation

110-210) in group B and 148 (range 125-170) in group $\mathrm{C}$. The $\chi^{2}$-test showed no statistically significant difference in the incidence of $\mathrm{AF}$ among patients with different AF prophylaxis ( $p>0.05$ ) (Tables 4 and 5 , Fig. 1). Analysis of variance (ANOVA) yielded no statistically significant difference among patients with different AF prophylaxis with respect to the highest ventricular frequency. Patients without prophylactic antiarrhythmic drugs had higher ventricular frequency during AF but this difference was not statistically significant ( $p>0.05$ ) (Table 6, Fig. 2). Analysis of correlation of particular risk factors with POAF yielded sta-

Table 10. Sex distribution of study patients

\begin{tabular}{|l|c|c|}
\hline Frequency table \\
\hline Sex & $\mathrm{n}$ & $\%$ \\
\hline Male & 389 & 66.0 \\
Female & 200 & 34.0 \\
\hline Total & 589 & 100.0 \\
\hline
\end{tabular}

Table 11. Sex and POAF incidence $(A F)$

\begin{tabular}{|l|c|c|c|}
\hline 2-way summary table: frequencies observed \\
\hline Sex & $\begin{array}{c}\mathrm{AF} \\
\text { yes }\end{array}$ & $\begin{array}{c}\mathrm{AF} \\
\text { no }\end{array}$ & Total \\
\hline $\begin{array}{l}\text { Male } \\
\%\end{array}$ & $\begin{array}{c}60 \\
15.42 \%\end{array}$ & $\begin{array}{c}329 \\
84.58 \%\end{array}$ & 389 \\
\hline Female & 17 & 183 & 200 \\
$\%$ & $8.50 \%$ & $91.50 \%$ & \\
\hline Total & 77 & 512 & 589 \\
\hline
\end{tabular}

$\mathrm{POAF}=$ postoperative atrial fibrillation; $\mathrm{AF}=$ atrial fibrillation

Table 12. Statistics: sex and POAF (AF)

\begin{tabular}{|l|c|c|c|}
\hline \multicolumn{4}{|l|}{ Statistics: $\operatorname{sex}(2) \times \mathrm{AF}(2)$} \\
\hline & $\chi^{2}$-test & $\mathrm{df}$ & $\mathrm{p}$ \\
\hline Pearson $\chi^{2}$-test & 5.573 & 1 & 0.018 \\
\hline
\end{tabular}

$\mathrm{POAF}=$ postoperative atrial fibrillation; $\mathrm{AF}=$ atrial fibrillation tistically significant correlation $(\mathrm{p}<0,05)$ for age $(\mathrm{Ta}-$ bles 7, 8 and 9), sex (Tables 10, 11 and 12), pneumonectomy versus other resections (Tables 13,14, 15 and 16), generalized atherosclerotic disease (in our study, it was defined as comorbidity including history of myocardial infarction or cerebrovascular insult, diagnosis of coronary disease and peripheral vascular disease) (Tables 17, 18 and 19), and ASA status (Tables 20, 21 and 22). Considering other states and comorbidities,

Table 13. Lung resection extent and $P O A F(A F)$

\begin{tabular}{|l|c|c|c|}
\hline \multicolumn{4}{|c|}{ 2-way summary table: frequencies observed } \\
\hline $\begin{array}{l}\text { Lung } \\
\text { resection }\end{array}$ & AF yes & AF no & Total \\
\hline PD & 3 & 23 & 26 \\
$\%$ & $11.54 \%$ & $88.46 \%$ & \\
\hline $\begin{array}{l}\text { PS } \\
\%\end{array}$ & 16 & 40 & 56 \\
\hline BID & $28.57 \%$ & $71.43 \%$ & \\
$\%$ & 2 & 25 & 27 \\
\hline BSD & $7.41 \%$ & $92.59 \%$ & \\
$\%$ & 3 & 13 & 16 \\
\hline LID & $18.75 \%$ & $81.25 \%$ & \\
$\%$ & 8 & 69 & 77 \\
\hline LSS & $10.39 \%$ & $89.61 \%$ & \\
$\%$ & 16 & 105 & 121 \\
\hline LIS & $13.22 \%$ & $86.78 \%$ & \\
\hline & 9 & 73 & 82 \\
\hline LSD & $10.98 \%$ & $89.02 \%$ & \\
\hline$\%$ & 16 & 133 & 149 \\
\hline LMD & $10.74 \%$ & $89.26 \%$ & \\
\hline$\%$ & 4 & 31 & 35 \\
\hline Total & $11.43 \%$ & $88.57 \%$ & \\
\hline & 77 & 512 & 589 \\
\hline
\end{tabular}

$\mathrm{POAF}=$ postoperative atrial fibrillation; $\mathrm{AF}=$ atrial fibrillation; $\mathrm{PD}$ = right pneumonectomy; $\mathrm{PS}=$ left pneumonectomy; $\mathrm{BID}=$ right inferior bilobectomy; $\mathrm{BSD}=$ right superior bilobectomy; $\mathrm{LID}=$ right inferior lobectomy; LSS = left superior lobectomy; LIS = left inferior lobectomy; $\mathrm{LSD}=$ right superior lobectomy; $\mathrm{LMD}=$ right middle lobe resection

Table 14. Statistics: lung resection extent and $P O A F(A F)$

\begin{tabular}{|l|c|c|c|}
\hline \multicolumn{4}{|c|}{ Statistics: lung resection $(9) \times \mathrm{AF}(2)$} \\
\hline & $\chi^{2}$-test & $\mathrm{df}$ & $\mathrm{p}$ \\
\hline Pearson $\chi^{2}$-test & 14.71278 & 8 & 0.06499 \\
\hline
\end{tabular}

$\mathrm{POAF}=$ postoperative atrial fibrillation; $\mathrm{AF}=$ atrial fibrillation 
Table 15. Pneumonectomies and $P O A F(A F)$

\begin{tabular}{|l|c|c|c|}
\hline \multicolumn{4}{|c|}{ 2-way summary table: frequencies observed } \\
\hline & $\begin{array}{c}\mathrm{AF} \\
\text { yes }\end{array}$ & $\begin{array}{c}\mathrm{AF} \\
\text { no }\end{array}$ & Total \\
\hline $\mathrm{PD}+\mathrm{PS}$ & 19 & 63 & 82 \\
$\%$ & $23.17 \%$ & $76.83 \%$ & \\
\hline Other & 58 & 449 & 507 \\
$\%$ & $11.44 \%$ & $88.56 \%$ & \\
\hline Total & 77 & 512 & 589 \\
\hline
\end{tabular}

$\mathrm{POAF}=$ postoperative atrial fibrillation; $\mathrm{AF}=$ atrial fibrillation; $\mathrm{PD}=$ right pneumonectomy; $\mathrm{PS}$ = left pneumonectomy

Table 16. Statistics: pneumonectomies and $P O A F$

\begin{tabular}{|l|c|c|c|}
\hline \multicolumn{4}{|l|}{ Statistics: PD + PS(2) $\times$ AF(2) } \\
\hline & $\chi^{2}$-test & df & p \\
\hline Pearson $\chi^{2}$-test & 8.547484 & 1 & 0.00346 \\
\hline
\end{tabular}

$\mathrm{POAF}=$ postoperative atrial fibrillation; $\mathrm{AF}=$ atrial fibrillation; $\mathrm{PD}=$ right pneumonectomy; $\mathrm{PS}=$ left pneumonectomy

\section{Table 17. Generalized atherosclerotic disease (GAD)}

\begin{tabular}{|l|c|c|}
\hline Frequency table: & \multicolumn{2}{|c|}{} \\
\hline GAD & $\mathrm{n}$ & $\%$ \\
\hline Yes & 78 & 13.2 \\
No & 511 & 86.8 \\
Total & 589 & 100.0 \\
\hline
\end{tabular}

Table 18. Generalized atherosclerotic disease and POAF

\begin{tabular}{|l|c|c|c|}
\hline 2-way summary table: frequencies observed \\
\hline GAD & $\begin{array}{c}\mathrm{AF} \\
\text { yes }\end{array}$ & $\begin{array}{c}\mathrm{AF} \\
\text { no }\end{array}$ & Total \\
\hline Yes & $\begin{array}{c}16 \\
\text { Row } \%\end{array}$ & $\begin{array}{c}62 \\
79.49 \%\end{array}$ & 78 \\
\hline No & 61 & 450 & 511 \\
Row $\%$ & $11.94 \%$ & $88.06 \%$ & \\
\hline Total & 77 & 512 & 589 \\
\hline
\end{tabular}

$\mathrm{GAD}=$ generalized atherosclerotic disease $\mathrm{POAF}=$ postoperative atrial fibrillation; $\mathrm{AF}=$ atrial fibrillation

Table 19. Statistics: generalized atherosclerotic disease and $P O A F$

\begin{tabular}{|l|c|c|c|}
\hline \multicolumn{3}{|l|}{ Statistics: $\mathrm{GAD}(2) \times \mathrm{AF}(2)$} \\
\hline & $\chi^{2}$-test & $\mathrm{df}$ & $\mathrm{p}$ \\
\hline Pearson $\chi^{2}$-test & 4.379084 & 1 & 0.03639 \\
\hline
\end{tabular}

$\mathrm{GAD}=$ generalized atherosclerotic disease $\mathrm{POAF}=$ postoperative atrial fibrillation; $\mathrm{AF}=$ atrial fibrillation
Table 20. ASA status

\begin{tabular}{|l|c|c|}
\hline \multicolumn{3}{|l|}{ Frequency table } \\
\hline ASA status & $\mathrm{n}$ & $\%$ \\
\hline$<$ ASA III & 237 & 40.2 \\
$\geq$ ASA III & 352 & 59.8 \\
Total & 589 & 100.0 \\
\hline
\end{tabular}

ASA status $=$ American Society of Anesthesiologists Physical Status

Table 21. ASA status and POAF incidence (FA)

2-way summary table: frequencies observed; marked cells had counts $>10$

\begin{tabular}{|l|c|c|c|}
\hline & AF & AF & Row \\
\hline$<$ ASA III & 18 & 219 & 237 \\
Row $\%$ & $7.59 \%$ & $92.41 \%$ & \\
\hline$\geq$ ASA III & 59 & 293 & 352 \\
Row $\%$ & $16.76 \%$ & $83.24 \%$ & \\
\hline Total & 77 & 512 & 589 \\
\hline
\end{tabular}

ASA status = American Society of Anesthesiologists Physical Status; $\mathrm{POAF}=$ postoperative atrial fibrillation; $\mathrm{AF}=$ atrial fibrillation

Table 22. Statistics: ASA status and POAF incidence (FA)

\begin{tabular}{|l|c|c|c|}
\hline \multicolumn{4}{|l|}{ Statistics: ASA status(2) x AF(2) } \\
\hline & $\chi^{2}$-test & df & p \\
\hline Pearson $\chi^{2}$-test & 10.47239 & 1 & 0.00121 \\
\hline
\end{tabular}

ASA status = American Society of Anesthesiologists Physical Status; $\mathrm{POAF}=$ postoperative atrial fibrillation; $\mathrm{AF}=$ atrial fibrillation

statistical analysis did not show statistical significance, e.g., thoracic epidural analgesia (Tables 23, 24 and 25), arterial hypertension (Tables 26, 27 and 28) and prior chemotherapy (Tables 29, 30 and 31), or the entities were too rare in our patients to be included in the analysis, e.g., diabetes mellitus, renal insufficiency and Teolin therapy.

\section{Discussion}

The search for the ideal antiarrhythmic for POAF prophylaxis is far from finished. Avoidance of $\beta$ blockade withdrawal in patients on chronic therapy with these medications is the simple and most effective preventive measure ${ }^{1,2,4}$. As with $\mathrm{AF}$ therapy, there is no ideal drug or method for POAF prevention in other patients. Magnesium supplementation is usually rec- 
Table 23. Thoracic epidural analgesia (TEA)

\begin{tabular}{|l|c|c|}
\hline Frequency table \\
\hline TEA & $\mathrm{n}$ & $\%$ \\
\hline Yes & 286 & 48.6 \\
No & 303 & 51.4 \\
\hline Total & 589 & 100.0 \\
\hline
\end{tabular}

Table 24. TEA and POAF (FA)

\begin{tabular}{|l|c|c|c|}
\hline 2-way summary table: frequencies observed \\
\hline TEA & $\begin{array}{c}\mathrm{AF} \\
\text { yes }\end{array}$ & $\begin{array}{c}\mathrm{AF} \\
\text { no }\end{array}$ & Total \\
\hline Yes & 36 & 250 & 286 \\
$\%$ & $12.59 \%$ & $87.41 \%$ & \\
\hline No & 41 & 262 & 303 \\
$\%$ & $13.53 \%$ & $86.47 \%$ & \\
\hline Total & 77 & 512 & 589 \\
\hline
\end{tabular}

TEA $=$ thoracic epidural analgesia; $\mathrm{POAF}=$ postoperative atrial fibrillation; $\mathrm{AF}=$ atrial fibrillation

Table 25. Statistics: TEA and POAF (FA)

\begin{tabular}{|l|c|c|c|}
\hline \multicolumn{4}{|l|}{ Statistics: TEA(2) $\mathrm{FA}(2)$} \\
\hline & $\chi^{2}$-test & $\mathrm{df}$ & $\mathrm{p}$ \\
\hline Pearson $\chi^{2}$-test & 0.1153593 & 1 & 0.73412 \\
\hline
\end{tabular}

$\mathrm{TEA}=$ thoracic epidural analgesia; $\mathrm{POAF}=$ postoperative atrial fibrillation; $\mathrm{AF}=$ atrial fibrillation

ommended ${ }^{1}$ and regularly administered in our patients. Some of the established antiarrhythmics such as amiodarone have questionable safety, especially in patients with pneumonectomy and COPD ${ }^{1,2,5}$. Diltiazem has been established as a prophylactic antiarrhythmic drug with moderate success ${ }^{1,2}$. It is recommended early postoperatively, especially in COPD patients, and is usually well tolerated ${ }^{3}$. Diltiazem is a nondihydropyridine calcium channel antagonist (class IV Vaughan Williams agent $)^{6}$. As AF prophylaxis, we administer it in oral form. In our study, we found no statistically significant difference between patient groups with and without diltiazem prophylaxis, suggesting that in our patients, POAF occurred as a complication of lung resection comparably often with and without diltiazem prophylaxis. So, we could not confirm the report by Amar et al. on diltiazem therapy and its significant
Table 26. Arterial bypertension

\begin{tabular}{|l|c|c|}
\hline Frequency table \\
\hline Arterial hypertension & $\mathrm{n}$ & $\%$ \\
\hline Yes & 272 & 46.2 \\
No & 317 & 53.8 \\
Total & 589 & 100.0 \\
\hline
\end{tabular}

Table 27. Arterial hypertension and POAF incidence

\begin{tabular}{|l|c|c|c|}
\hline \multicolumn{4}{|c|}{ 2-way summary table: frequencies observed } \\
\hline AH & $\begin{array}{c}\mathrm{AF} \\
\text { yes }\end{array}$ & $\begin{array}{c}\mathrm{AF} \\
\text { no }\end{array}$ & Total \\
\hline Yes & 40 & 232 & 272 \\
$\%$ & $14.71 \%$ & $85.29 \%$ & \\
\hline No & 37 & 280 & 317 \\
$\%$ & $11.67 \%$ & $88.33 \%$ & \\
\hline Total & 77 & 512 & 589 \\
\hline
\end{tabular}

$\mathrm{AH}=$ arterial hypertension; $\mathrm{POAF}=$ postoperative atrial fibrillation; $\mathrm{AF}=$ atrial fibrillation

Table 28. Statistics: arterial hypertension and POAF

\begin{tabular}{|l|c|c|c|}
\hline \multicolumn{3}{|l|}{ Statistics: $\mathrm{AH}(2) \times \mathrm{AF}(2)$} \\
\hline & $\chi^{2}$-test & $\mathrm{df}$ & $\mathrm{p}$ \\
\hline Pearson $\chi^{2}$-test & 1.185774 & 1 & 0.27619 \\
\hline
\end{tabular}

$\mathrm{AH}=$ arterial hypertension; $\mathrm{POAF}=$ postoperative atrial fibrillation; $\mathrm{AF}=$ atrial fibrillation

POAF rate reduction ${ }^{3}$. Our speculation that patients with diltiazem prophylaxis generally have somewhat slower ventricular response during POAF (median 150; without any prophylaxis, median 160), which could have some protective properties per se, was not confirmed by statistical analysis. Maybe the most problematic part of the study was the uneven dosage of diltiazem postoperatively, especially on day 0; also, underdosing was probably quite common due to hemodynamic instability of patients at that time. However, Hiran et al. ${ }^{2}$ in their guidelines suggest that diltiazem prophylaxis per os be initiated in recovery room at a dose of 30-60 mg every 6 hours, depending on the patient related factors and with "hold parameters", and we did so accordingly. There is also the issue of the oral route of taking the drug and its gastrointestinal resorption in the early postoperative course. In our study, the incidence of POAF was $13.1 \%$, which is consistent 
Table 29. Prior chemotherapy

\begin{tabular}{|l|c|c|}
\hline Frequency table \\
\hline CHT & $\mathrm{n}$ & $\%$ \\
\hline Yes & 127 & 21.6 \\
No & 462 & 78.4 \\
Total & 589 & 100.0 \\
\hline
\end{tabular}

$\mathrm{CHT}=$ chemotherapy

\section{Table 30. Prior chemotherapy and POAF incidence}

2-way summary table: frequencies observed; marked cells had counts $>10$

\begin{tabular}{|l|c|c|c|}
\hline CHT & $\begin{array}{c}\text { AF } \\
\text { yes }\end{array}$ & $\begin{array}{c}\text { AF } \\
\text { no }\end{array}$ & Total \\
\hline Yes & 14 & 113 & 127 \\
$\%$ & $11.02 \%$ & $88.98 \%$ & \\
\hline $\begin{array}{l}\text { No } \\
\%\end{array}$ & 63 & 399 & 462 \\
& $13.64 \%$ & $86.36 \%$ & \\
\hline Total & 77 & 512 & 589 \\
\hline
\end{tabular}

$\mathrm{POAF}=$ postoperative atrial fibrillation; $\mathrm{AF}=$ atrial fibrillation; $\mathrm{CHT}=$ chemotherapy

Table 31. Statistics: prior chemotherapy and POAF incidence

\begin{tabular}{|l|c|c|c|}
\hline \multicolumn{4}{|l|}{ Statistics: CHT(2) $\mathrm{AF}(2)$} \\
\hline & $\chi^{2}$-test & $\mathrm{df}$ & $\mathrm{p}$ \\
\hline Pearson $\chi^{2}$-test & 0.5984023 & 1 & 0.43919 \\
\hline
\end{tabular}

$\mathrm{POAF}=$ postoperative atrial fibrillation CHT $=$ chemotherapy

with literature data ${ }^{2}$, where it is reported to be $12 \%$ $44 \%$. If we exclude pneumonectomies, then our POAF incidence was $11.4 \%$. As most of our patients were transferred from the ICU on postoperative day 2, we can speculate that there were much more unnoticed and asymptomatic POAF episodes on surgical ward. We confirmed literature reports on patient age, sex, pneumonectomy (especially intrapericardial and extrapleural extensions) and generalized atherosclerotic disease (i.e. coronary disease) as risk factors ${ }^{1,2}$ (statistically significant results). Some other risk factors could not be confirmed due to the relatively low number of affected patients in our sample (hyperthyroidism, cardiac diseases other than coronary disease, arrhythmias, diabetes mellitus, etc. $)^{1,2}$. We believe that there were more patients with these risk factors, but it was not recorded in their medical documentation. It should be noted that we found a statistically significant correlation between ASA status and POAF, but not between epidural analgesia/anesthesia and POAF. ASA status, as stated in the literature, correlates with perioperative mortality "even better than other classifications of mortality and morbidity"7,8. TEA is considered to exert some protective effect against $\mathrm{POAF}^{9}$. Arterial hypertension was not confirmed as a risk factor for POAF, in contrast to the 2014 American Heart Association Atrial Fibrillation Guidelines and relevant literature ${ }^{1,2,4}$. Preoperative chemotherapy, indicated as a risk factor for postoperative cardiovascular events ${ }^{10}$, does not seem to increase the risk of POAF. To establish more definitive conclusions, additional study in a bigger sample and including more and better defined risk factors is needed.

\section{References}

1. Frendel G, Sodickson AC, Chung MK, et al. 2014 AATS guidelines for the prevention and management of perioperative atrial fibrillation and flutter for thoracic surgery procedures. J Thorac Cardiovasc Surg. 2014;148:153-93, http://dx.doi.org/ 10.1016/j.jtcvs.2014.06.036

2. Hiran CF, Jaklitsch MT, Walsh GL, et al. The Society of Thoracic Surgeons practice guideline on the prophylaxis and management of atrial fibrillation associated with general thoracic surgery: executive summary. Ann Thorac Surg. 2011;92: 1144-52. http://dx.doi.org/10.1016/j.athoracsur.2011.06.104

3. Amar D, Roistacher N, Rusch VW, et al. Effects of diltiazem prophylaxis on the incidence and clinical outcome of atrial arrhythmias after thoracic surgery. J Thorac Cardiovasc Surg. 2000;120:790-8 http://dx.doi.org/10.1067/mtc.2000.109538

4. January CT, Wann LS, Alpert JS, et al. 2014 AHA/ACC/HRS Guideline for the Management of Patients with Atrial Fibrillation: Executive Summary. A Report of the American College of Cardiology/American Heart Association Task Force on Practice Guidelines and the Heart Rhythm Society. J Am Coll Cardiol. 2014;64(21):2246-80, https://dx.doi.org/10.1161/ CIR.0000000000000040

5. Van Mieghem W, Coolen L, Malysse I, Lacquet LM, Deneffe GJ, Demedts MG. Amiodarone and the development of ARDS after lung surgery. Chest. 1994;105:1642-5, http://dx. doi.org/10.1378/chest.105.6.1642

6. Echizen H, Eichelbaum M. Clinical pharmacokinetics of verapamil, nifedipine and diltiazem. Clin Pharmacokinet. 1986; 11:425-49, https://dx.doi.org/10.2165/00003088-198611060$-00002$

7. Sidi A, Lobato EB, Cohen JA. The American Society of Anesthesiologists' Physical Status: category V revisited. J Clin Anes- 
th. 2000;12(4):328-34, http://dx.doi.org/10.1016/S0952-8180 (00)00168-9

8. Wolters U, Wolf T, Stutzer H, Schroder T. ASA classification and perioperative variables as predictors of postoperative outcome. Br J Anesth. 1996;77:217-22, https://doi.org/10.1093/ $\mathrm{bja} / 77.2 .217$

9. Hanna MN, Murphy JD, Kumar K, Wu CI. Regional techniques and outcome: what is the evidence? Curr Opin Anaes- thesiol. 2009;22(5):672-7, https://dx.doi.org/ 10.1097/ACO. 0b013e32832f330a

10. Ferguson MK, Saha-Chaudhuri P, Mitchel JD, Varela G, Brunelli A. Prediction of major cardiovascular events after lung resection using a Modified Scoring System. Ann Thorac Surg. 2014;97:1135-41, http://dx.doi.org/10.1016/j.athoracsur.2013. 12.032

Sažetak

\section{PROFILAKSA POSLIJEOPERACIJSKE ATRIJSKE FIBRILACIJE I RESEKCIJA PLUĆA - NAŠA ISKUSTVA SA 608 UZASTOPNIH BOLESNIKA}

\section{Karadža, D. Stančić-Rokotor, J. Špiček Macan, N. Hodoba, N. Kolarić i S. Sakan}

Poslijeoperacijska atrijska fibrilacija je česta komplikacija resekcije pluća. Ona dovodi do povišenog pobola i smrtnosti, produljenog bolničkog liječenja i povećane potrošnje sredstava u torakokirurških bolesnika. U skladu s tim, savjetuje se neki oblik farmakološke profilakse. U naših bolesnika odabrani lijek je kalcijev antagonist diltiazem. Skupili smo podatke o svih 608 bolesnika podvrgnutih resekciji pluća (ne manjoj od lobektomije) u razdoblju između studenog 2012. i svibnja 2015. Ovo razdoblje uključuje bolesnike koji su primali diltiazem tijekom njihova poslijeoperacijskog boravka u Jedinici intenzivnog liječenja i na kirurškom odjelu te bolesnike koji ga nisu primali. Bolesnici s atrijskom fibrilacijom prije operacije i bolesnici s elektrostimulatorom srca isključeni su iz obrade. Ostali bolesnici podijeljeni su u tri skupine: skupinu liječenu nekim antiaritmikom prije operacije koji su nastavili svoju terapiju i nakon operativnog zahvata, skupinu na profilaksi diltiazemom i skupinu bez profilakse. Skupljeni podaci su statistički analizirani. Nismo našli statistički značajnu razliku između skupina u incidenciji poslijeoperacijske atrijske fibrilacije $(\mathrm{p}<0,05)$.

Ključne riječi: Diltiazem; Atrijska fibrilacija; Torakalna kirurgija 